\title{
AUSTRIAN TRANSITION TO OPEN ACCESS (AT2OA) - EIN ÜBERBLICK
}

\author{
von Brigitte Kromp, Maria Seissl und Tobias Zarka
}

Zusammenfassung: Im Rahmen des Hochschulraumstrukturmittelprojekts „Austrian Transition to Open Access" (AT2OA) sollen durch Neugestaltung der Lizenzverträge mit den Anbietern und durch gezielte Publikationsunterstützung der Forschenden eine Steigerung des österreichischen Open Access-Publikationsoutputs generiert und neue Wege für das Open Access-Publizieren eröffnet werden. In vier Teilprojekten werden unterschiedliche Maßnahmen zur Unterstützung dieses Transformationsprozesses bearbeitet. Ergänzt werden diese Teilprojekte durch Vernetzungsaktivitäten und Maßnahmen zum Wissenstransfer. Der Artikel gibt einen kurzen Überblick über die inhaltlichen Schwerpunkte und die Governance des Projektes.

Schlagwörter: Hochschulraumstrukturmittelprojekt; Austrian Transition to Open Access (AT2OA); Governance; Open Access; Open Access Transformation; Open Access Transition; Bundesministerium für Bildung, Wissenschaft und Forschung

\section{AUSTRIAN TRANSITION TO OPEN ACCESS (AT2OA) - IN A NUTSHELL}

Abstract: The goal of the Higher Education Area Structural Funds project "Austrian Transition to Open Access" (AT2OA) is to support the large-scale transformation of scientific publications from Closed to Open Access, and to implement measures supporting this initiative. The project aims to increase Austria's Open Access publication output by restructuring licence agreements with publishers and by providing targeted support for researchers' publication activities. It will also establish new venues for Open Access publishing. The subprojects are complemented by networking and knowledge transfer activities. The article gives a brief overview of the main topics and the governance of the project.

Keywords: Higher Education Area Structural Funds Project; Austrian Transition to Open Access (AT2OA); Governance; Open Access; Open Access Transformation; Open Access Transition; Austrian Federal Ministry of Education, Science and Research

DOI: https://doi.org/10.31263/voebm.v72i1.2274 
(c) Brigitte Kromp, Maria Seissl, Tobias Zarka

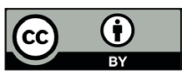

Dieses Werk ist lizenziert unter einer

Creative-Commons-Lizenz Namensnennung 4.0 International

Das Projekt Austrian Transition to Open Access (AT2OA) ${ }^{1}$ hat das Ziel, die Transformation von Closed zu Open Access bei wissenschaftlichen Publikationen zu unterstützen und voranzutreiben ${ }^{2}$. Von Jänner 2017 bis voraussichtlich Dezember 2020 arbeiten die 21 öffentlichen Universitäten Österreichs und drei weitere kooptierte Partnereinrichtungen an der Umsetzung des Kooperationsprojektes.

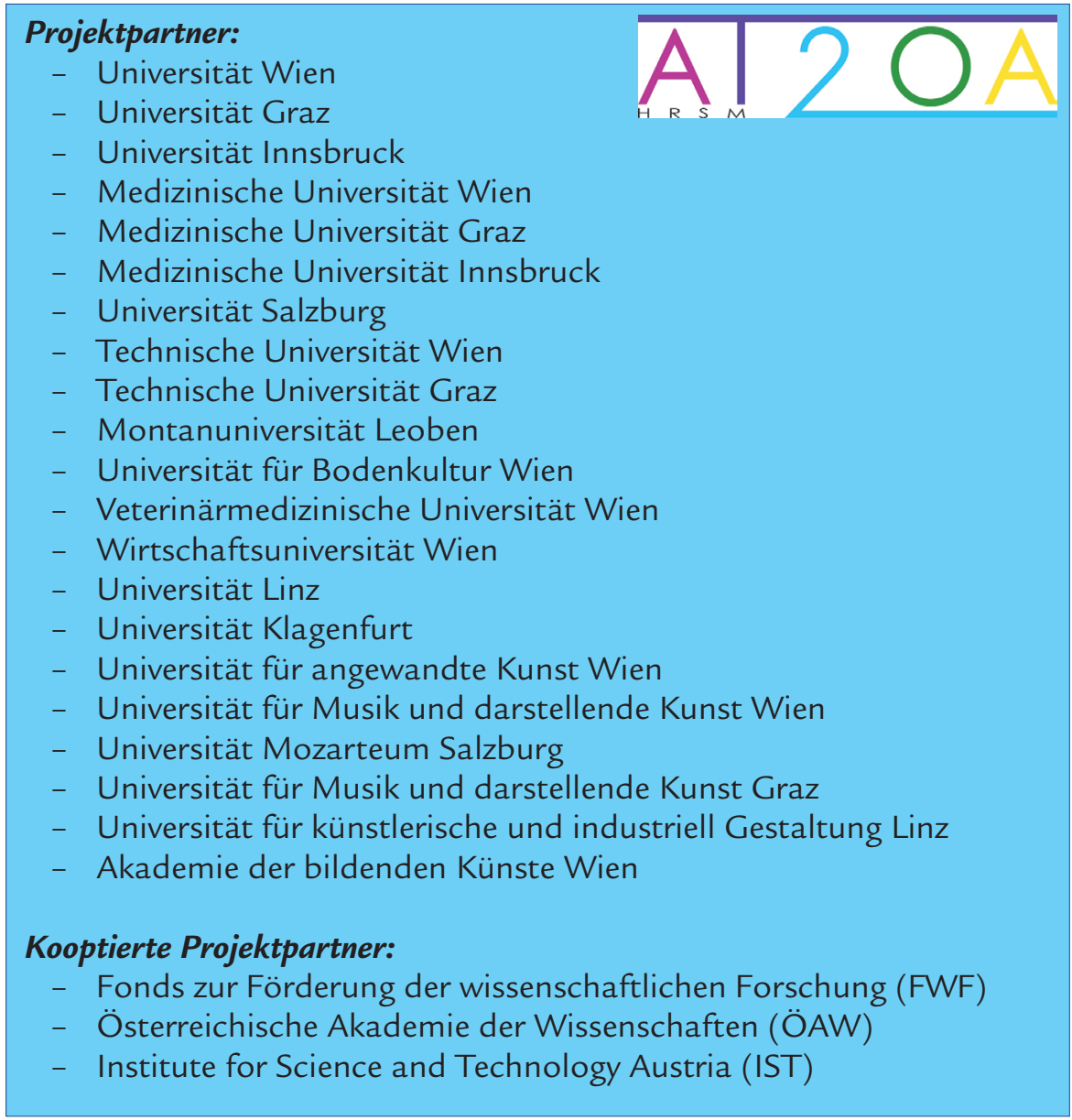




\section{Inhaltliche Schwerpunkte}

In vier Teilprojekten werden die Themen

- „Analyse der Auswirkung einer Umstellung auf Open Access“ (TP1),

- „Finanzierung von Open Access-Übergangsmodellen“ (TP2),

- „Auf-, Ausbau und Finanzierung von OA-Publikationsfonds“ (TP3),

- „Förderung von OA-Publikationen und alternativen OA-Publikationsmodellen" (TP4)

von zahlreichen MitarbeiterInnen der Partnereinrichtungen bearbeitet. Ergänzt werden diese Teilprojekte durch Vernetzungsaktivitäten und Maßnahmen zum Wissenstransfer.

Im Rahmen des ersten Teilprojekts wird untersucht, ob für Österreich eine vollständige Umstellung vom bisherigen Zeitschriftenfinanzierungsmodell (Subskriptionsmodell) auf Open Access ohne Mehrkosten möglich ist und welche Auswirkungen eine Umstellung auf die (Literatur)Budgets und Zahlungsflüsse in Österreich haben wird. Darüber hinaus wird auf Basis von internationalen Best-Practice-Modellen ein Konzept für das Monitoring des Open Access-Publikationsoutputs in Österreich entwickelt.

Das zweite Teilprojekt ist vor allem mit der Verhandlung und dem Abschluss von Verträgen mit Open Access-Komponenten sowie deren Evaluation beschäftigt.

Teilprojekt drei unterstützt den Auf- und Ausbau lokaler Publikationsfonds in operativer und finanzieller Weise. Ziel ist es hierbei, die Nachhaltigkeit lokaler Fonds zu sichern, die Transparenz zu erhöhen und die Förderrichtlinien in einigen zentralen Punkten unter strategischen Gesichtspunkten zu vereinheitlichen.

Um der ständig steigenden Marktkonzentration entgegenzuwirken, werden im vierten Teilprojekt alternative Publikationsmodelle an den Universitäten initiiert. So soll z.B. ein Anstoß für universitäre und österreichweite Open Access-Initiativen von Universitätsverlagen und universitätsnahen Verlagen gegeben und die Förderung von Open Access-Monographien und Qualitätssicherungskomponenten in diesem Bereich vorangetrieben werden.

\section{Projektstruktur}

Im gesamten Projekt arbeiten mehr als 60 Personen überwiegend aus den Universitätsbibliotheken, zum Teil aber auch aus anderen universitären Abteilungen zusammen. Diese Mitarbeiterlnnen leisten gemeinsam mit den 
zwei Mitarbeitern im Koordinationsbüro des Projektes den größten Teil des fachlichen Inputs in den vier Teilprojekten. Durch diese breite Beteiligung wird sichergestellt, dass möglichst viele Interessen der verschiedenen Stakeholdergruppen - z.B. publikationsschwache versus publikationsstarke, geisteswissenschaftliche versus naturwissenschaftliche - berücksichtigt werden.

Die strategische Begleitung wird von einem Lenkungskomitee, bestehend aus zehn Rektorlnnen und Vizerektorlnnen, und der Projektleitung übernommen, sodass eine möglichst rasche und direkte Abstimmung zu essentiellen Fragen des Projektes zwischen den Leitungsebenen der Universitäten und den operativ handelnden Personen aus den betreffenden Bibliotheken gewährleistet wird. Um die gewonnenen Erkenntnisse möglichst breit zu verteilen, gibt es eine Vollversammlung, die aus Vertreterlnnen aller beteiligten Institutionen besteht und regelmäßig über die Fortschritte des Projektes informiert wird.

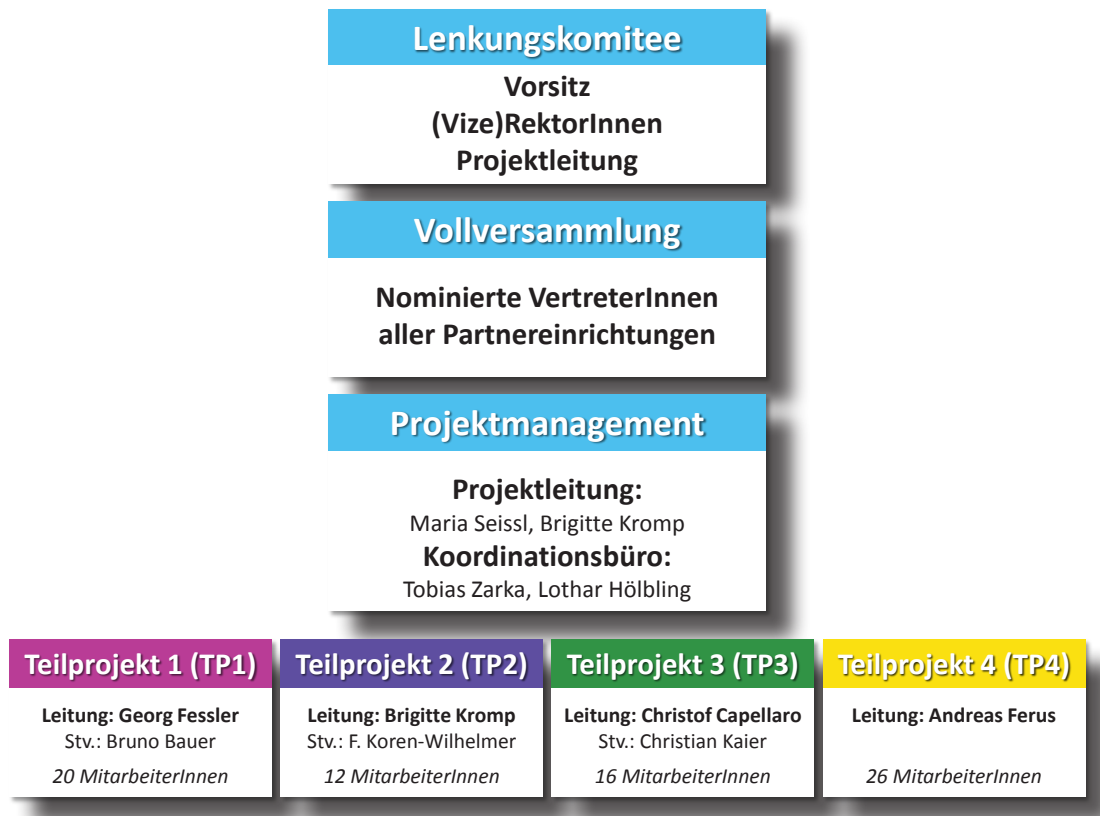

Abb. 1: Gliederung des Hochschulraumstrukturmittelprojektes Austrian Transition to Open Access (AT2OA) (Grafik: Tobias Zarka)

Das Projektmanagement, bestehend aus Projektleitung und Koordinationsbüro, ist an der Universität Wien angesiedelt und fungiert als Knoten- 
punkt für das gesamte AT2OA-Netzwerk. Die Leitungen der Teilprojekte teilen sich die Mitglieder. Das erste Teilprojekt verantworten die Wirtschaftsuniversität Wien und die Medizinische Universität Wien, das zweite die Universität Wien und die Universität Graz, für Teilprojekt drei haben sich die Universität Salzburg und die Universität Graz verpflichtet, für Teilprojekt vier die Akademie der bildenden Künste Wien.

Die große Anzahl von Mitarbeiterlnnen, die vielfältigen und raschen Entwicklungen im Bereich Open Access sowie die umfangreichen und teilweise komplexen Ergebnisse der Arbeit der vier Teilprojekte machen die Kommunikation sowohl innerhalb des Projekts als auch die Organisation der Informationsflusses nach außen zu einem ganz wichtigen Teil der Arbeit des Koordinationsbüros.

\section{Kommunikationsstruktur}

Der Großteil der Kommunikation innerhalb des Projektes findet via E-Mail statt. Hierzu wurde für jedes der vier Teilprojekte eine Service-E-Mailadresse eingerichtet, die eingehende Nachrichten an alle am jeweiligen Teilprojekt beteiligten Personen weiterleitet.

Eine eigene Projekt-Webseite (www.at2oa.at) sorgt einerseits neben grundlegenden Informationen über das Projekt auch für Updates zu AT2OA-bezogenen Publikationen und Veranstaltungen, welche für alle einsehbar sind. Darüber hinaus dient ein zusätzlicher, geschlossener Bereich zur Ablage und Einsichtnahme von Protokollen und sonstigen internen Dokumenten, die lediglich für Projektmitarbeiterlnnen bestimmt sind. Konzeption, Programmierung und Gestaltung der Webseite obliegt dem Koordinationsbüro.

Weiters organisiert jede der vier Teilprojektleitungen selbstständig mehrmals im Jahr Treffen, bei denen die jeweiligen Mitarbeiterlnnen gemeinsam die einzelnen Deliverables erarbeiten. Bis dato (Stand März 2019) fanden bereits über 40 solcher Arbeitsgruppen-Treffen statt. Zusätzlich wird von der Gesamt-Projektleitung noch etwa vierteljährlich ein Treffen der Teilprojektleitungen einberufen sowie zumindest einmal im Jahr eine Vollversammlung, zu der alle nominierten Vertreterlnnen der Partnereinrichtungen eingeladen werden. Die Zusammenarbeit mit dem Lenkungskomitee erfolgt durch mindestens jährlich stattfindende Treffen mit den nominierten (Vize)Rektorlnnen bzw. durch Information des gesamten Forums Forschung und Erschließung der Künste der Österreichischen Universitätenkonferenz. 


\section{Resümee}

Nach mehr als der Hälfte der Laufzeit kann eine durchaus positive Bilanz gezogen werden:

Durch die breite Beteiligung von unterschiedlichsten Interessensgruppen, wie allen öffentlichen Universitäten, dem bedeutendsten Forschungsförderer im Bereich der Grundlagenforschung, dem FWF (Fonds zu Förderung der wissenschaftlichen Forschung), der Österreichischen Universitätenkonferenz, Forschungseinrichtungen und der KEMÖ (Kooperation E-Medien Österreich) wird ein tragfähiges Netzwerk zur Diskussion und Zusammenarbeit in vielen Punkten der vielschichtigen und komplexen Open Access-Landschaft aufgebaut.

Der von den Mitgliedern in TP1 bereits im März 2019 abgelieferte Abschlussbericht zur Transition-Studie ${ }^{3}$ liefert umfangreiches Datenmaterial zu Budget- und Kostenfragen eines weiteren Ausbaus von Open Access. Gemeinsam mit den im Rahmen von TP2 abgeschlossenen transformativen Verträgen mit Open Access-Komponenten ${ }^{4}$ führt das dazu, dass Österreich eines der europäischen Länder mit der höchsten Anzahl an Plan S kompatiblen Verlagsabkommen ist. Die Zusammenarbeit der Universitäten in TP3 liefert nicht nur Richtlinien zu einer abgestimmten Vorgangsweise $^{4}$ im Bereich von Förderungen im Rahmen von Open Access-Fonds, sondern ergibt auch ganz neue Einsichten und Ansätze in Bezug auf die Finanzierung von Open Access-Publikationen in Hinsicht auf die Globalbudgets der Universitäten. Und letztendlich werden durch die intensive Arbeit an unterschiedlichsten Ideen zu alternativen Open AccessPublikationsmodellen ${ }^{6}$ in TP4 neue OA-Publikations-Projekte initiiert.

Insgesamt hat AT2OA bereits in der Hälfte der Laufzeit zu einem vertieften Verständnis über Veränderungen im Bereich der wissenschaftlichen Kommunikation durch Open Access geführt und die Projektpartner sowohl mögliche Hindernisse als auch Chancen erkennen lassen. Zur ersten Information aber auch für ein umfassenderes Bild der Aktivitäten und gewonnenen Erfahrungen eignet sich auf alle Fälle immer ein Blick auf die AT2OA-Webseite. 
Mag. ${ }^{a}$ Brigitte Kromp

ORCID: https://orcid.org/0000-0002-7793-3453

Universität Wien, Bibliotheks- und Archivwesen

E-Mail: brigitte.kromp@univie.ac.at

Mag. ${ }^{\mathrm{a}}$ Maria Seissl

Universität Wien, Bibliotheks- und Archivwesen

E-Mail: maria.seissl@univie.ac.at

Tobias Zarka

Universität Wien, Bibliotheks- und Archivwesen

E-Mail: tobias.zarka@univie.ac.at

1 Austrian Transition to Open Access (AT2OA): https://www.at2oa.at/

2 Bruno Bauer, Christof Capellaro, Andreas Ferus, Georg Fessler, Renata Granat, Lothar Hölbling, Christian Kaier, Frank Koren-Wilhelmer, Brigitte Kromp, Maria Seissl, Tobias Zarka (2018): Austrian Transition to Open Access (AT2OA). Bibliothek Forschung und Praxis 42(3), 463-475. https://doi.org/10.1515/bfp2018-0062

3 Georg Fessler \& Lothar Hölbling (2019). Ausbau von Open Access an den österreichischen Universitäten: Budgetärer Mehrbedarf für die Jahre 2019-2021. Zenodo. http://doi.org/10.5281/zenodo.2621015

4 Kooperation E-Medien Österreich (12.02.2018): Wiley, Kooperation E-Medien Österreich und FWF geben kombinierte Open-Access Publikations- und Subskriptions-Vereinbarung bekannt. https://www.konsortien.at/meldungen-Details.asp? meldungenid $=848$

5 Handreichung Open Access-Pubikationsfonds: http://doi.org/10.5281/ zenodo. 2653725

6 Falk Reckling \& Andreas Ferus (2019). Die Förderung von alternativen, nicht-kommerziellen Open Science-Infrastrukturen \& -Services (OSIS) durch Forschungseinrichtungen in Österreich - Empfehlungen, Kriterien \& Modelle (Version 1.1). Zenodo. http://doi.org/10.5281/zenodo. 2549452 